\title{
After COACT trial-new perspectives for the management of non-ST elevation myocardial infarction: early versus late cardiac catheterization post cardiac arrest
}

\author{
Miguel A. Maturana ${ }^{1}$, Charles F. Clinton ${ }^{2}$, Selene Caballero-Cummings ${ }^{3}$, Brandon Cave ${ }^{4}$, Amal Khan ${ }^{5}$, \\ Amit Nanda ${ }^{1}$, Devarshi Ardeshna ${ }^{1}$, Joel Raja ${ }^{1}$, Rami N. Khouzam ${ }^{6}$ \\ ${ }^{1}$ Department of Internal Medicine, University of Tennessee Health Science Center, Memphis, TN, USA; ${ }^{2}$ Department of Cardiology, Universidad \\ de Costa, San Jose, Costa Rica; ${ }^{3}$ Department of Medicine, Universidad Autonoma de Chihuahua, Chihuahua, Mexico; ${ }^{4}$ Department of Pharmacy, \\ Methodist University Hospital, Memphis, TN, USA; ${ }^{5}$ Department of Medicine, Dow University of Health Sciences, Pakistan; ${ }^{6}$ Department of \\ Internal Medicine, Division of Cardiovascular Diseases, University of Tennessee Health Science Center, Memphis, TN, USA \\ Contributions: (I) Conception and design: MA Maturana, RN Khouzam; (II) Administrative support: MA Maturana, RN Khouzam; (III) Provision of \\ study materials or patients: All authors; (IV) Collection and assembly of data: MA Maturana, CF Clinton, S Caballero-Cummings, B Cave, A Khan, A \\ Nanda, D Ardeshna, JM Raja; (V) Data analysis and interpretation: MA Maturana, CF Clinton, S Caballero-Cummings, B Cave, A Khan, A Nanda, \\ D Ardeshna, JM Raja; (VI) Manuscript writing: All authors; (VII) Final approval of manuscript: All authors. \\ Correspondence to: Miguel A. Maturana, MD. Department of Internal Medicine, University of Tennessee Health Science Center, 288 Night Sail Drive \\ South, Apt 316, Memphis, TN, USA. Email: mmaturan@uthsc.edu.
}

\begin{abstract}
Out-of-hospital cardiac arrest (OHCA) is the leading cause of death in the United States, as $90 \%$ of them are fatal per the 2018 American Heart Association statistics. As many as fifty-percent of cardiac arrest events display an initial rhythm of pulseless ventricular tachycardia (pVT) and ventricular fibrillation (VF), and of those, coronary artery disease (CAD) is found in $60-80 \%$ of patients. Following return of spontaneous circulation, patients who present with ST-elevation myocardial infarction (STEMI) should undergo an early invasive strategy and primary intervention, which is well-established guideline-based management. The support of such a strategy in patients suspected to have underlying cardiac cause but without ST-elevation has been waxing and waning in the literature. The Coronary Angiography after Cardiac Arrest (COACT) trial was designed to compare survival between an immediate or delayed coronary angiography strategy in non-STEMI (NSTEMI) OHCA patients, following successful resuscitation. We present a systematic review of the history of management strategies in OHCA and propose guidelines to manage such patients in light of the COACT trial.
\end{abstract}

Keywords: Non-ST elevation myocardial infarction (NSTEMI); out-of-hospital cardiac arrest (OHCA); Coronary Angiography after Cardiac Arrest trial (COACT trial); cardiac arrest; cardiac catheterization

Submitted Jun 30, 2019. Accepted for publication Jul 29, 2019.

doi: $10.21037 /$ atm.2019.08.12

View this article at: http://dx.doi.org/10.21037/atm.2019.08.12

\section{Introduction}

Out-of-hospital cardiac arrest (OHCA) is the leading cause of death, occurring in approximately 350,000 Americans annually in the United States (1). Pulseless ventricular tachycardia $(\mathrm{pVT})$ and ventricular fibrillation $(\mathrm{VF})$ are the initial rhythm in almost $25-50 \%$ of these patients (1-4). Coronary artery disease (CAD) is found in $60-80 \%$ of these patients and only $10 \%$ of these patients survive to hospital discharge $(1,2,5)$. While current guidelines not only recommend immediate coronary angiography with percutaneous coronary intervention (PCI) in patients who present with ST-elevation myocardial infarction (STEMI) and OHCA (class I indication, level of evidence B), they also support the use in OHCA believed to be mediated by a cardiac 
cause (class IIa, level of evidence B). However, the survival benefit in OHCA without evidence of STEMI is still unclear (6).

Large cohort trials have suggested increased survival to discharge in OHCA presenting with $\mathrm{pVT}$ or $\mathrm{VF}$ when treated with angiography and PCI (2,7-9). During the past 15-20 years, there has been a shift in clinical practice towards early angiography as studies showed that almost $70 \%$ patients with OHCA have CAD and over half of them have an occluded coronary artery (10). Several studies showed that angiographically documented acute coronary syndrome (ACS) was believed to precede OHCA in $40-85 \%$ patients and successful PCI in these patients was associated with reduced mortality and beneficial outcomes including better neurological recovery (10-13). Observational studies and meta-analysis have supported the potential benefit of early angiography and PCI in OHCA in the absence of STEMI (11-13). However, they have unanimously suggested the need of randomized trials to validate their findings. Also, these and contemporaneous trials describe the underutilization of angiography and PCI in OHCA due to the high mortality in this subset, predominantly resulting from the heightened sensitivity to publicly reported mortality and outcome data (14-16). Randomized trials comparing the outcomes of the two strategies in non-STEMI (NSTEMI) OHCA patients have long been recommended.

\section{Current guidelines}

According to American College of Cardiology (ACC)/ American Heart Association (AHA) Guidelines, reperfusion therapy should be performed to all eligible patients with STEMI and ischemic symptoms of less than 12 hours of duration, preferably via PCI (17). Similar recommendations are suggested by the European Society of Cardiology (18). Standard of care in patients with NSTEMI requires risk stratification to determine treatment with either an ischemia-guided strategy or early invasive strategy (PCI or CABG) (19). Per 2014 ACC/AHA guidelines, urgent/ immediate ( $\leq 2$ hours) invasive strategy of diagnostic angiography with revascularization is indicated in NSTEMI patients who have refractory angina, electrical or hemodynamic instability. An early ( $\leq 24$ hours) invasive strategy is indicated in stable NSTEMI patients who have an elevated risk for clinical events (GRACE score $>140$ ). For patients that are not at intermediate or high risk (diabetes mellitus, renal insufficiency with GFR $<60 \mathrm{~mL} / \mathrm{min} / 1.73 \mathrm{~m}^{2}$, reduced LV systolic function with $\mathrm{EF}<0.40$, early postinfarction angina, PCI within 6 months, prior CABG,
GRACE score of 109-140, and TIMI score $\geq 2$ ) a delayed invasive approach is reasonable (19).

The strongest degree of evidence in current American Guidelines to favor early compared to delayed invasive strategy in patients with NSTEMI, currently relies on observational studies, randomized control trials and a meta-analysis. The ICTUS trial enrolled 1,200 patients with NSTEMI who were randomized to an early invasive strategy or to a more conservative (selectively invasive) strategy. This study did not show that an early invasive strategy was superior to a selectively invasive for the primary composite endpoint of reducing death $(\mathrm{P}=0.49)$ or spontaneous MI $(\mathrm{P}=0.20)$ (20). Same question was also assessed by a meta-analysis that pooled data from 11 trials [7 randomized controlled trials (RCTs) and 4 observational studies]. Analysis of the RCTs was inconclusive for survival benefit of early versus delayed invasive strategy [OR: 0.83 (95\% CI: 0.64 to 1.09); $\mathrm{P}=0.180$ ], and there were no significant differences in MI or major bleeding (21). A similar result was found with the observational studies.

Current available evidence does not allow firm conclusions to be drawn in favor or against an early invasive approach in the NSTEMI population. Data are limited by the small sample size of the individual trials, low event rates, inconsistency in timing of intervention, and heterogenous patient profiles (19). A more definitive RCT, properly powered for mortality as a single end-point, and related cost-effective analyses are warranted to quantify the potential survival benefits and assess the feasibility of an early approach in patients with NSTEMI (21).

Similarly, for patients with return of spontaneous circulation following an OHCA, the first step in management may vary depending on ECG findings. For those without ST-segment elevation, current guidelines are unclear and thus physicians find themselves in the dreaded gray area of medicine. Should the patient be rushed for an immediate coronary angiography? Or, should neurological recovery be given precedence over cardiac evaluation? What parameters justify the decision to delay? What is the risk versus benefit ratio in either strategy? The answers to these questions are just beginning to be answered, namely in a newly published Coronary Angiography after Cardiac Arrest (COACT) trial.

\section{COACT}

The COACT trial was an investigator-initiated, randomized, multicenter trial conducted from January 2015 to July 
2018. Enrollment included 552 patients who experienced an OHCA with an initial shockable rhythm and were unconscious after the return of spontaneous circulation. Patients were randomized in a 1:1 fashion to a strategy of immediate coronary angiography, and PCI if necessary, compared to a strategy of delayed angiography with respect to overall survival. On analysis, the patient population was a fair representation of the general population. Both arms were well matched in regard to their baseline characteristics.

The trial concluded that there was no statistically significant difference between a strategy of immediate angiography as compared to a strategy of delayed angiography in the primary endpoint of survival at 90 days (odds ratio, $0.89 ; 95 \% \mathrm{CI}, 0.62$ to $1.27 ; \mathrm{P}=0.51$ ) (22). Furthermore, patients in COACT who died were more than three times as likely to die from neurologic injury than from a cardiac cause. These findings suggest that in patients without STEMI, perhaps one can defer coronary angiography and allow for neurological and clinical recovery to be the initial focus. This may allow for earlier implementation of postcardiac arrest protocols and once the patient is more stable, cardiac catheterization can be considered.

COACT, although it should be appreciated as the first randomized clinical trial investigating this clinical conundrum, is not without its limitations. First, when conducting a clinical, investigators seem not to have taken into consideration the symptoms and signs preceding cardiac arrest. These could have aided in differentiating non-cardiac from cardiac causes and would have allowed for a more refined study. Second, the physicians were not blind to the assigned groups, inviting the potential for bias in patient management. Third, there were several factors that did not correlate well with the real-life management of cardiac arrests. In COACT, a vast majority of OHCAs were witnessed $(79.9 \%$ in the immediate angiography group, $76.6 \%$ in the delayed angiography group), the median time from arrest to basic life support was reported as 2 minutes and the median time from arrest to return of spontaneous circulation was reported as 15 minutes (22). These conditions represent a very limited number of patients when compared to real-life situations, and thus question the external validity of the study. Fourth, acute thrombotic occlusions were more than twice as prevalent in the delayed coronary angiography group (7.6\%) as compared to the early coronary angiography group (3.4\%). This difference could have favored the results against a strategy of early coronary angiography. Fifth, COACT incorporated data from patients managed across 19 individual centers, which could have differed significantly with regards to how the patients were managed. Thus, there may have been multiple modifying factors that could not be considered for analysis. Sixth, a potential confounding variable to consider was one of the secondary outcomes, time to achieve target temperature. A statistically significant difference was seen between the two groups, with those in the delayed angiography group achieving target temperature within 4.7 hours, as compared to 5.4 hours in the early angiography group (effect size, 1.19, 95\% CI: 1.04 to 1.36) (22). Finally, it should be noted that in patients older than 70 years and a prior history of CAD, immediate angiography did show a statistically significant survival benefit over delayed angiography (23). So, in a subset of patients perhaps early angiography may be warranted.

\section{Discussion}

The conflicting results for adequate timing for cardiac catheterization in patients with NSTEMI have led to the development of multiple new randomized clinical trials. As stated by Yannopoulos et al., "although randomized clinical trials are planned, or ongoing, current scientific evidence rests principally on observational case series with their potential confounding selection bias." (24). Most of these new studies are designed with a stronger level of evidence compared to previous observational trials.

The ACCESS trial (25), sponsored by the University of Minnesota, is a randomized, open label, interventional trial, currently underway with expected completion in June 2020. Target enrollment is 864 patients to assess neurological outcome in patients undergoing initial cardiac catheterization versus conservative management after achieving ROSC without evidence of STEMI in admission ECG. Unique to ACCESS is enrollment criteria does not specify neurologic status for inclusion, while COACT restricted to a Glasgow Coma Scale of $<8$ after ROSC.

The DISCO-2 trial (26) is an open-label, randomized, multicenter clinical trial conducted in Sweden and sponsored by the Swedish Heart and Lung Foundation and Laerdal Foundation for Acute Medicine. With a total of 1,006 patients enrolled, the DISCO-2 trial will be the largest clinical trial to date, more than twice the size of COACT. This trial, will evaluate the results of immediate coronary angiography after OHCA on 30-day survival in patients with NSTEMI. DISCO-2 does not restrict initial rhythm to $\mathrm{pVT}$ or $\mathrm{VF}$, as was required in COACT. Additionally, endpoint assessments will evaluate 30-day 
outcomes and long-term outcomes of neurological recovery, cognitive function and cardiac function at 6 months.

The EMERGE trial (27), is a prospective, randomized, open label, multicenter clinical trial based in France and sponsored by Hôspitaux de Paris. This trial began recruitment in January 2017 with expected completion in July 2019 with a target enrollment of 970 patients. In EMERGE, the primary endpoint is survival rate with no or minimal neurological sequalae at 6 months of early cardiac catheterization versus late cardiac catheterization in patients with OHCA and NSTEMI. Inclusion criteria includes patients aged 18 or older without an obvious cause of noncardiac arrest. In EMERGE, late cardiac catheterization is defined as greater than 48 hours, while in COACT late catheterization was defined after neurological recovery.

\section{Conclusions}

In conclusion, for patients with return of spontaneous circulation following an OHCA and non-ST-segment elevation, present guidelines are unclear. Sadly, for far too long physicians have struggled with this gray area of medicine, questioning whether to proceed with urgent or delayed coronary angiography. Thus far, current American guidelines have had to rely on data from observational studies and a meta-analysis from three trials: FRISC-II, ICTUS, and RITA-3. As of March 2019, AHA reported: "there is no consensus about the value and necessity of early catheterization for resuscitated patients without ST-segment elevation." (24). Being the first randomized, controlled trial, COACT has added new energy to the discussion. It concluded that a strategy of immediate angiography was not better than a strategy of delayed angiography with respect to overall survival at 90 days. However, it is still too early to say if and when guidelines will change, as more data is surely needed. We look to the future with more trials are on the way, most notably the ACCESS, DISCO-2 and EMERGE trials. We are hopeful that one day physicians will look back and see the COACT study in having helped pave the road to clearer, evidence-based guidelines.

\section{Acknowledgments}

None.

\section{Footnote}

Conflicts of Interest: Dr. Cave discloses that he serves on the
Speakers Bureau for Portola pharmaceuticals. The other authors have no conflicts of interest to declare.

Ethical Statement: The authors are accountable for all aspects of the work in ensuring that questions related to the accuracy or integrity of any part of the work are appropriately investigated and resolved.

\section{References}

1. Mozaffarian D, Benjamin EJ, Go AS, et al. Heart disease and stroke statistics--2015 update: a report from the American Heart Association. Circulation 2015;131:e29-322.

2. Vyas A, Chan PS, Cram P, et al. Early coronary angiography and survival after out of-hospital cardiac arrest. Circ Cardiovasc Interv 2015;8:e002321.

3. Geri G, Dumas F, Bougouin W, et al. Immediate percutaneous coronary intervention is associated with improved short- and long-term survival after outof-hospital cardiac arrest. Circ Cardiovasc Interv 2015;8:e02303.

4. Graham R, McCoy MA, Schultz AM, et al. Institute of Medicine Committee on the Treatment of Cardiac Arrest. Current Status and Future Directions: Strategies to Improve Cardiac Arrest Survival: A Time to Act. Washington, DC: National Academic Press, 2015:363-398.

5. Kern KB, Lotun K, Patel N, et al. INTCAR-Cardiology Registry. Outcomes of comatose cardiac arrest survivors with and without ST-segment elevation myocardial infarction: importance of coronary angiography. JACC Cardiovasc Interv 2015;8:1031-40.

6. Callaway CW, Donnino MW, Fink EL, et al. Part 8: Postcardiac arrest care: 2015 American Heart Association guidelines update for cardiopulmonary resuscitation and emergency cardiovascular care. Circulation 2015;132:S465-82.

7. Cronier P, Vignon P, Bouferrache K, et al. Impact of routine percutaneous coronary intervention after out-of hospital cardiac arrest due to ventricular fibrillation. Crit Care 2011;15:R122.

8. Reynolds JC, Callaway CW, El Khoudary SR, et al. Coronary angiography predicts improved outcome following cardiac arrest: propensity-adjusted analysis. J Intensive Care Med 2009;24:179-86.

9. Wijesekera VA, Mullany DV, Tjahjadi CA, et al. Routine angiography in survivors of out of hospital cardiac arrest with return of spontaneous circulation: a single site registry. BMC Cardiovasc Disord 2014;14:30. 
10. Spaulding CM, Joly LM, Rosenberg A, et al. Immediate coronary angiography in survivors of out-of-hospital cardiac arrest. N Engl J Med 1997;336:1629-33.

11. Dankiewicz J, Nielsen N, Annborn M, et al. Survival in patients without acute ST elevation after cardiac arrest and association with early coronary angiography: a post hoc analysis from the TTM trial. Intensive Care Med 2015;41:856-64.

12. Hollenbeck RD, McPherson JA, Mooney MR, et al. Early cardiac catheterization is associated with improved survival in comatose survivors of cardiac arrest without STEMI. Resuscitation 2014;85:88-95.

13. Khan MS, Shah SMM, Mubashir A, et al. Early coronary angiography in patients resuscitated from out of hospital cardiac arrest without ST-segment elevation: a systematic review and meta-analysis. Resuscitation 2017;121:127-34.

14. Waldo SW, McCabe JM, O'Brien C, et al. Association between public reporting of outcomes with procedural management and mortality for patients with acute myocardial infarction. J Am Coll Cardiol 2015;65:1119-26.

15. Moscucci M. Public reporting of percutaneous coronary intervention outcomes: harm or benefit? J Am Coll Cardiol 2015;65:1127-9.

16. Rab T, Kern KB, Tamis-Holland JE, et al. Interventional Council, American College of Cardiology. Cardiac arrest: a treatment algorithm for emergent invasive cardiac procedures in the resuscitated comatose patient. J Am Coll Cardiol 2015;66:62-73.

17. O'Gara PT, Kushner FG, Ascheim DD, et al 2013 ACCF/ AHA guideline for the management of ST-elevation myocardial infarction: a report of the American College of Cardiology Foundation/American Heart Association Task Force on Practice Guidelines. Circulation 2013;127:e362-425.

18. Ibanez B, James S, Agewall S, et al 2017 ESC Guidelines for the management of acute myocardial infarction in patients presenting with ST-segment elevation: The Task Force for the management of acute myocardial infarction in patients presenting with ST-segment elevation of the European Society of Cardiology (ESC). Eur Heart J

Cite this article as: Maturana MA, Clinton CF, CaballeroCummings S, Cave B, Khan A, Nanda A, Ardeshna D, Raja J, Khouzam RN. After COACT trial-new perspectives for the management of non-ST elevation myocardial infarction: early versus late cardiac catheterization post cardiac arrest. Ann Transl Med 2019;7(17):413. doi: 10.21037/atm.2019.08.12
2018;39:119-77.

19. Amsterdam EA, Wenger NK, Brindis RG, et al 2014 AHA/ACC Guideline for the Management of Patients with Non-ST-Elevation Acute Coronary Syndromes: a report of the American College of Cardiology/American Heart Association Task Force on Practice Guidelines. J Am Coll Cardiol 2014;64:e139-228.

20. Damman P, Hirsch A, Windhausen F, et al. 5-year clinical outcomes in the ICTUS (Invasive versus Conservative Treatment in Unstable coronary Syndromes) trial a randomized comparison of an early invasive versus selective invasive management in patients with non-STsegment elevation acute coronary syndrome. J Am Coll Cardiol 2010;55:858-64.

21. Navarese EP, Gurbel PA, Andreotti F, et al. Optimal timing of coronary invasive strategy in non-ST-segment elevation acute coronary syndromes: a systematic review and meta-analysis. Ann Intern Med 2013;158:261-70.

22. Lemkes JS, Janssens GN, van Royen N. Coronary Angiography after Cardiac Arrest without ST-Segment Elevation. Reply. N Engl J Med 2019;381:189-90.

23. Lemkes JS, Janssens GN, van der Hoeven NW, et al. Coronary angiography after cardiac arrest without STsegment elevation. N Engl J Med 2019;380:1397-407.

24. Yannopoulos D, Bartos JA, Aufderheide TP, et al. The evolving role of the cardiac catheterization laboratory in the management of patients with out-of-hospital cardiac arrest: a scientific statement from the American Heart Association. Circulation 2019;139: e530-52.

25. Yannopoulos D, Aufderheide T. ACCESS to the Cardiac Cath Lab in Patients Without STEMI Resuscitated From Out-of-hospital VT/VF Cardiac Arrest. Available online: https://clinicaltrials.gov/ct2/show/NCT03119571

26. Lagedal R, Elfwén L, James S, et al. Design of DISCODirect or Subacute Coronary Angiography in Out-ofHospital Cardiac Arrest study. Am Heart J 2018;197:53-61.

27. EMERGEncy Versus Delayed Coronary Angiogram in Survivors of Out-of-hospital Cardiac Arrest. Available online: https://clinicaltrials.gov/ct2/show/NCT02876458 